\title{
Critical Analysis of the Role of Micronutrient - Zinc on Covid-19 Activity
}

\author{
Mohammadreza Mohseni ${ }^{1}$, Mahsa Rastegar Moghaddam Mansouri ${ }^{2}$, Sivakumar J T Gowder ${ }^{3,4 *}$ \\ ${ }^{1}$ Lifestyle Gym, Tehran, Iran \\ ${ }^{2}$ Faculty of Sports Sciences, Hakim Sabzevari University, Sabzevar, Iran \\ ${ }^{3}$ Bionanotechnology Research Group, Ton Duc Thang University, Ho Chi Minh City, Vietnam
}

${ }^{4}$ Faculty of Pharmacy, Ton Duc Thang University, Ho Chi Minh City, Vietnam

*Corresponding author: Sivakumar J T Gowder, Ton Duc Thang University, Ho Chi Minh City, Vietnam

\section{ARTICLE INFO \\ Received: 幽 September 22, 2020 \\ Published: September 29, 2020}

Citation: Mohammadreza Mohseni, MahsaRastegar Moghaddam Mansouri, Sivakumar J T Gowder. Critical Analysis of the Role of Micronutrient - Zinc on Covid-19 Activity. Biomed J Sci \& Tech Res 30(5)-2020. BJSTR. MS.ID.005007.

\author{
ABSTRACT
}

Coronavirus disease 2019 (COVID-19) is a third highly pathogenic coronavirus that reported first in China and in three months of time, nearly $3.5 \%$ out of 79,968 cases died due to this viral disease. This virus similar to previous ones has receptors in host body that is angiotensin converting enzyme 2 (ACE2), which has similar acts like carboxypeptidase and cleaves the C-terminal ester bond of peptide. ACE2 is a zinccontaining metalloprotease that remove the amino acid residue from the $\mathrm{C}$-terminal of peptide chain. Metalloprotease catalyzes those mechanisms which involve the metal. Most of the metalloproteases require zinc for their function as a cofactor. Treatment with chelating agents leads to complete inactivation of the metalloproteases. Although zinc is an immunomodulator, but here it is suggested that zinc enhances the activity of COVID-19 that needs further investigation.

\begin{abstract}
Abbreviations: COVID-19: Coronavirus Disease 2019; ACE2: Angiotensin Converting Enzyme 2; MERS-CoV: Middle East Respiratory Syndrome Corona Virus; RER: Rough Endoplasmic Reticulum; ORFs: Open Reading Frames; S: Spike; E: Envelope; N: Nucleocaspids; M: Membrane; ER: Endoplasmic Reticulum; ERAD: Endoplasmic Reticulum Associated Degradation; PvY: Polyomavirus; UPR: Unfolded Protein Response; MAMs: Mitochondria-Associated-Membranes; ROS: Reactive Oxygen Spices; IRE1: Inositol Requiring Enzyme 1; PKR: Protein Kinase RNA-Activated; ATF: Activating Transcription Factor; BiP: Binding Immunoglobulin Protein; MAPK: Mitogen-Activated Protein Kinase; TNFR1: Tumor Necrosis Factor Receptor 1; TNF- $\alpha$ : Tumor Necrosis Factor-Alpha; JNK: . c-Jun N-Terminal Kinase; RBD: Receptor Binding Domain; RAS: Renin-Angiotensin System; Ang: Angiotensin; C-Terminal: Carboxy-Terminal; CPA: Carboxypeptidase A
\end{abstract}

\section{Introduction}

Coronavirus disease 2019 (COVID-19) is the third highly pathogenic coronavirus after SARS-CoV and middle east respiratory syndrome corona virus (MERS-CoV). It has been reported that from the December 2019 that the first COVID-19 appeared in China till the first day of March 2020 and about 79,968 cases were reported. $3.5 \%$ of them were dead and $52 \%$ of them were cured. Though there are 35,414 cases under way, but they were not reported to the public. Coronavirus has a single-stranded RNA which classified into positive or negative based on polarity of viral RNA. Coronavirus has a positive sense viral RNA genome ranging from 26 to 32 kilobases in length that acts as mRNA and translated into protein in host cell. It is an enveloped virus that has an outer wrapping or envelope which comes from the infected cell, or host, in a process called "budding off." While in the process of budding formation, a small 
piece of plasma membrane of cell has "enveloped" or wrapped into an outer coat of the newly formed viral particles [1,2].

In this virus, the rough endoplasmic reticulum (RER) makes the coronavirus envelope. There are four types of coronavirus such as $\alpha, \beta, \gamma$, and $\delta$ that the current virus classified in $\beta$ group. COVID-19 genome contains about ten open reading frames (ORFs), which the first of ORFs are about $75 \%$ of RNA length and translated into two large polyproteins $1 \mathrm{a}$ and $1 \mathrm{ab}$ (pp1a, pp1ab) and following translation, they proceed into 16 non-structural proteins (nsp1 to nsp16) and form the replicase transcriptase complex. Replicase is as enzyme that catalyzes RNA synthesis in the presence of a template of RNA, and on the other hand transcriptase is an enzyme that catalyzes the formation of RNA from DNA in transcription process. It has been shown that nsp 16 could modify coronavirus mRNAs (3); nsp's arrange new membrane and create doublemembrane vesicles from the hosted cell-RER for virus replication and transcription. Other 25\% of COVID-19 ORFs genome has four structural proteins as spike (S), envelope (E), nucleocaspids $(\mathrm{N})$, and membrane (M) (Figure 1). The N protein holds the RNA genome and the rest structural proteins create viral envelope. The $\mathrm{S}$ protein is responsible to attach virus to host cell which is explained in detail as follow [1-3].

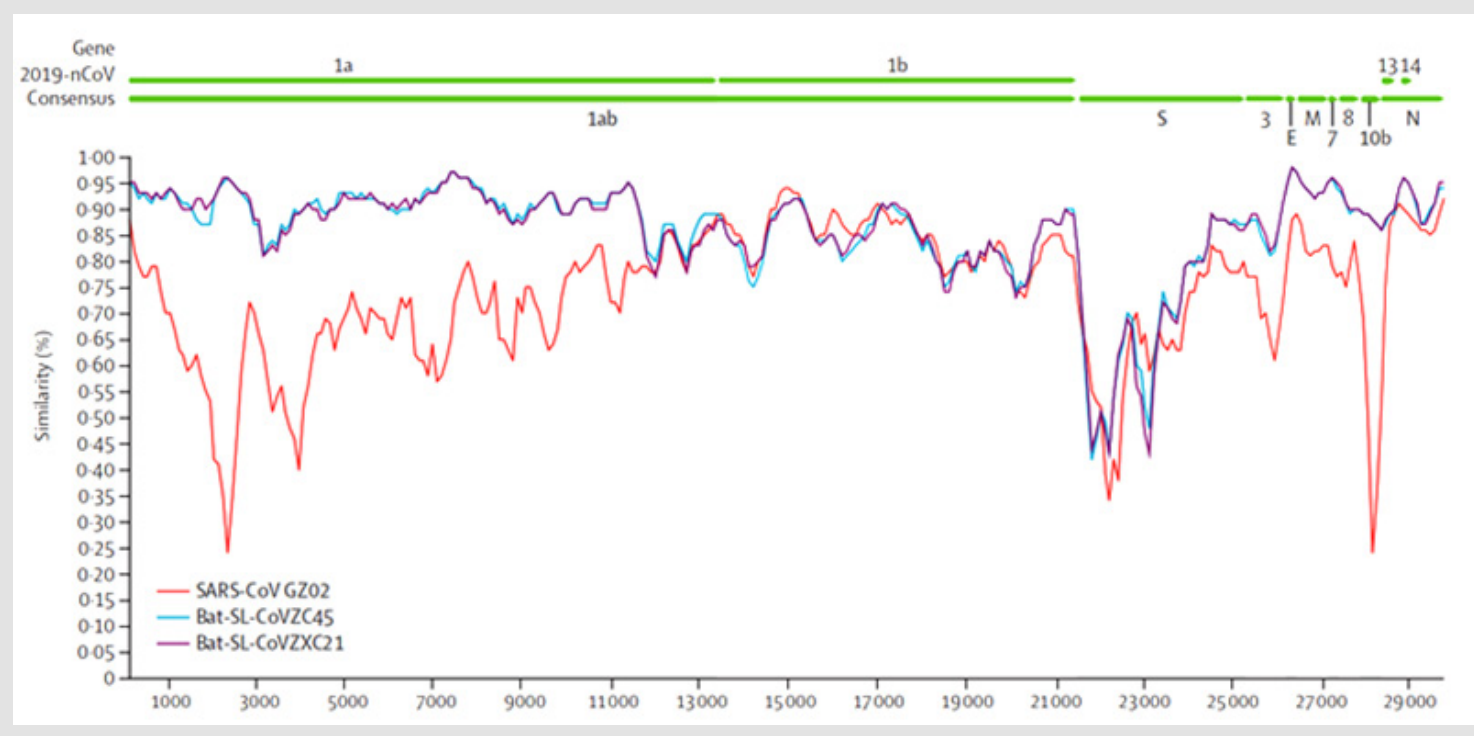

Figure 1: COVID-19 genome contains about ten open reading frames (ORFs), of which,the first of ORFs are about 75\% of RNA and translate into two large polyproteins 1a and 1ab (pp1a, pp1ab). The other 25\% of COVID-19 ORFs genome have four structural proteins as spike (S), envelope (E), nucleocapsid $(\mathrm{N})$, and membrane $(\mathrm{M})$. In this figure a sequence identity between the consensus of 2019-nCoV and representative betacoronavirus genomes is also shown. As it is figured the similarity in ppla between current coronavirus and previous forms have shown (2).

The S protein or envelope spike glycoprotein of COVID-19 directly latches onto human cells, undergoes structural changes and could fuse to cell membrane and enter into host cells. It has two subunits as S1 and S2. The former one has RBD and bind to host receptor (that is angiotensin converting enzyme 2 or ACE2) and the later subunit fuse the virus and host cell membrane [4]. Then, the gene of COVID-19 enter host cell, its RNA genome releases in cytoplasm and translates into two polyproteins and structural proteins. Following this phase, its genome is copied and formed into new glycoproteins that insert into endoplasmic reticulum membrane and form nucleocapsid. Capsid is a protein shell of the virus that consists several structural subunits which made of protomers and capsid is near to genetic material of virus. Viral particles enter endoplasmic reticulum (ER), use its double membrane and create viral vesicles which could fuse to cell membrane [5].To better understand the virus functions in the body, the interaction between the virus and ER as a crucial organelle to support virus entry, replication and assembly is to be explained.

Virus needs a proper complex membranous network to reside in the host cell, and hence, plasma, endolysosomal, and ER are appropriate candidate organelles in this process. Each of these viruses could act through one of them as suitable membrane system. Each virus could latch to its special triggers which are located on the membranous system inside a cell. Receptors, proteases, chaperons are some examples of cellular triggers. ER, which is formed of a group of flattened sacs emerged as a continuous membrane system within the cytoplasm of cells. It performed varied functions such as synthesis and folding in addition to modifications and transport of proteins [5].Virus per se manipulates host immune system to survive and using the host cell-organelles, the membrane provides a gold chance for virus to reside. ER works in two way-directions, 
firstly translates and folds new synthesis-proteins through COPII complex and transfers them to Golgi and secondly could misfold protein by ER associated degradation (ERAD) system (ERAD is endogenous ER quality control system) and translocate them to cytosol to be degraded via ubiquitin-proteasome pathway. Virus could use ER properties to enter the host cell cytosol.

Each virus needs to pass a barrier to enter host cell. There are three barriers which could be plasma, endolysosome, or ER. Most viruses use first two barriers to enter host cell and only polyomavirus (PyV) family could penetrate ER. Polymaviruses meaning "many tumours" and are small nonenveloped doublestranded DNA viruses which its-induced illness occur in time of immune system weakness, especially $\mathrm{T}$ cell deficiency. Nonenveloped DNA viruses are composed of capsid protein and nucleic acid, consists of DNA or RNA [5-7]. As it has mentioned above, coronavirus replication similar to polyomavirus needs ER cellular compartment. Coronavirus infection causes ER stress and unfolded protein response (UPR). ER is a major store of calcium and is classified into two or three groups based on appearance or membrane structure, respectively. Appearance classification is based on presence (rough ER) or absence (smooth ER) of ribosome on the ER membrane. Membrane structure classification is based on curvature of the membrane that consists of nuclear envelope, sheet-like cisternae, and tubules which the later one has the highest membrane curvature [6].

ER acts as the site of protein synthesis, calcium regulation and storage, lipid synthesis and storage, and glucose metabolism. Also, it acts like a bridge between other intracellular organelles, connects with mitochondria via mitochondria-associatedmembranes (MAMs) that has a vital irrefutable role in calcium homeostasis, attaches with plasma membrane through proteins like stromal interaction molecule 1 and calcium release-activated calcium channel protein 1 that is worked by calcium concentration. ER simply folds about one-third of cellular proteins and when its capacity to fold them becomes saturated, it is called ER stress that can be caused by impaired disulfide bond formation, or over expression or even mutation in protein entering pathway. Providing oxidizing environment is necessary for disulfide bond formation if oxidation and its induced-reactive oxygen spices (ROS) exceed the ER antioxidant capacity, the ER stress happens. ER and ROS connection is mediated through various signaling pathways such as calcium signaling [6].

UPR is another ER stress that is activated in response to an accumulation of unfolded proteins in the ER lumen. UPR initially aims to restore ER homeostasis and while it fails, it activates three sensors or branches through them could modulate wide variety of signaling pathways and results in apoptosis. Those branches that are located in the ER luminal consists of inositol requiring enzyme 1 (IRE1), protein kinase RNA-activated (PKR)-like ER kinase (PERK) and activating transcription factor 6 (ATF6). Under normal situation, these branches bound with chaperone, heat shock protein A5, and binding immunoglobulin protein (BiP) which keeps those sensors or branches inactive. By accumulating misfolded proteins, BiP engages which results in releasing all three branches which modulate and activate various signaling pathways such as mitogenactivated protein kinase (MAPK), autophagy, apoptosis, and innate immune response $[3,6]$. Under a constant ER stress, different cell death pathways run through the cell that could be intrinsic (mitochondrial) or even extrinsic (death receptor).

Also, all three UPR branches are activated in apoptosis. Activation of death receptors which locate on plasma membrane or release cytochrome $\mathrm{c}$ from mitochondria run caspase cascade. Both IRE1 and PERK induce apoptosis in response to ER stress. Necroptosis is one kind of cell death that works with receptorinteracting protein kinase 1 (RIPK1) and RIPK3 and linked to ER stress which is activated via tumor necrosis factor receptor 1 (TNFR1) while apoptosis is locked. TNFR1 is a receptor that binds with tumor necrosis factor-alpha (TNF- $\alpha$ ) that is a cell signaling cytokine and involve in inflammation. Autophagic cell death similar to necroptosis is run while apoptosis is blocked and could mediate non-apoptotic cell death [6]. ER stress and consequence-UPR contribute to viral replication during coronavirus infection. It has been reported that SARS-CoV virus results in ER stress and upregulate and engage $\mathrm{BiP}$ in infected cells, which results in released and active branches and run ER apoptosis-signaling pathway [3]. Under stress cell activates adaptive mechanism to control stress and regain ER homeostasis that intensity and duration of stress that have direct effects on the mechanism [6].

Following the prolong stress and UPR activation, cell cannot regain its homeostasis and switch cell to death. The IRE1 as one of the UPR branch induce apoptosis through JNK pathway. c-Jun $\mathrm{N}$-terminal kinase (JNK) pathway is one of the major pathways of the MAPK signaling pathway. PERK as another UPR branch is vital for attenuating of protein synthesis in ER stress. The N-terminal of PERK is homologous to IRE1. Activated-PERK results in activating transcription factor 4 (ATF4), which could run a set of stress response genes and express C/EBP homologous protein (CHOP) as an endoplasmic reticulum stress-inducible protein. CHOP as a transcription factor and a direct target of ATF4, increases ER calcium, promote expression of apoptotic genes, and play a critical role in the regulation of programmed cell death. Under normal situation, CHOP over expression cannot run cell death that is shown cell death needs additional signaling such as ATF4 [6,8]. It has been reported that PERK and ATF4 pathways are activated in some corona virus and play pro-apoptotic function that can be studied in detail elsewhere.

Nuclear factor kappa-light-chain-enhancer of activated B cells that is known as NF-kB, is a transcription factor and is considered as a regulator of pro-inflammatory factors such as IL-6, IL-8, or TNF- $\alpha$ which per se overexpresses the S protein of SARS CoV. Under 
normal environment, inhibitor of NF-kB alpha (Ik-B $\alpha$ ) controls and limits the NF-kB activation in cytoplasm (3). Coronavirus leads to ER stress that phosphorylates eukaryotic initiation factor $2 \alpha$ (eIF $2 \alpha$ ) which it acts in the early steps of protein synthesis. PhosphorylatedeIF $2 \alpha$ decreases Ik-B $\alpha$ which consequently increases the NF-kB, induces IL-6, IL-8, TNF- $\alpha$, and over-expresses S protein of SARS-CoV [3]. The $S$ protein or envelope spike glycoprotein consists receptor binding domain (RBD) that binds virus to its receptor that is named angiotensin-converting enzyme 2 (ACE2) on host cell. The S protein of current COVID-19 has considerable homology with SARS-CoV, but there are still differences between current virus and previous one which explain why SARS-CoV antibodies are mostly invalidate for the last version of this virus $[4,9]$.

The S proteins of COVID-19 and SARS-CoV have remarkable homology. It has been reported that new SARS virus $S$ protein and its RBD could recognize human ACE2 more efficiently and has strong binding to it which makes this virus more contagious [10]. The S protein of COVID-19 is strong case of study as it is a target for development vaccine due to its important roles in viral attachment to human cells [4]. Renin-angiotensin system (RAS) is a signaling pathway that regulates blood pressure, natriuresis (large amount of sodium in urine), and blood volume. Its activity is high in lung. Angiotensin I (Ang I) under the effects of dipeptide carboxypeptidase and ACE metabolize and change to angiotensin II (Ang II). AngII under the effects of carboxypeptidase and ACE2 metabolize to angiotensin 1-7 (Ang 1-7) which works as vasodilator. AngII works as blood pressure regulator, sodium and water homeostasis regulator, control neurohumoral system; also, it produces reactive oxygen spices (ROS) and leads to vascular cells apoptosis and acts as main factor in hypertension-induced tissue damage.ACE2 is a type 1 integral membrane glycoprotein and express in almost all tissues specially lung, kidney, endothelium, and heart.

A single transmembrane sequence is located in the integral membrane protein. We can notice a single transmembrane sequence in integral membrane protein. Type -1 membrane proteins have a cleavable $\mathrm{N}$-terminal signal sequence with a transmembrane sequence that normally situated nearer to C-terminus. ACE2 is important to regulate local levels of Ang II and Ang 1-7. Interestingly, blocking renin-angiotensin pathway decreases the SARS-CoV induced-lung injury and ACE2 expression has direct effects on intensity of virus-injury. In contrast, it has been reported that RAS inhibition might up-regulate ACE2 and thus increase the COVID-19 outcomes [11-13]. ACE2 has a extracellular domain that contain a single catalytic metallopeptidase unit that share considerable similarity with catalytic domain of ACE, but the activity of ACE2 is not stop by ACE inhibitor. ACE2 metallopeptidase unit consists metal ion on its active site that is mostly zinc $[11,13]$. Matrix metallopeptidase or metalloproteinase is a calciumdependent zinc-containing endopeptidase (any of a group of enzymes that hydrolyze peptide bonds within the long chains of protein molecules) that degrades extracellular matrix proteins and plays crucial roles in cell proliferation, differentiation, and apoptosis [14].

ACE2 acts like carboxypeptidase. Carboxypeptidase is a protease enzyme that hydrolyzes (cleave) a peptide bond at the carboxy-terminal (C-terminal) end of protein or peptide. In contrast, aminopeptidase acts different and opposite of carboxypeptidase and cleaves peptide bond from $\mathrm{N}$-terminal of protein [11]. Carboxypeptidase A cleaves the C-terminal ester bond of peptide or depsipeptidase that have a free $\mathrm{C}$-terminal carboxyl group. Carboxypeptidase A (CPA) is a zinc-containing metalloprotease that remove the amino acid residue from the $\mathrm{C}$-terminal of peptide chain. Metalloprotease is a protease enzyme that catalyzes that mechanism which involves metal. Most metalloprotease require zinc as a cofactor. Metal ion is integrated to the protein through three legends. The ligands which coordinating metal ion can vary with histidine, lysine, glutamate, aspartate, arginine.Fourth coordination position is taken up by a labile water molecule. Treatment with chelating agents leads to complete inactivation of metalloprotease. Chelating agents are organic compounds that have metal ions to form complex ring like structures called as chelates. Heterocyclic compounds are compounds that have atoms of atleast two different elements [15-17].

Zinc, as a micronutrient has different roles in body, and it acts like signaling molecule and its homeostasis is precisely regulated by zinc transporters. Zinc is a cofactor, works as a second messenger like calcium, effects on enzymes functions, and acts as transcription factor. Its deficiency causes atrophy, impairs immune system, emotional disorders, weight loss, neurosensory dysfunctions and if it is not treated, the patient would have to die within years [18]. Zinc, as it has been reported, is an immune system essential component which also is important for ACE2 metallopeptidase domain that consists of it on its active site. It is estimated that increased zinc-consumption could increase COVID-19 activity by elevating its receptor's function (ACE2 function) on host cell. In this case, zinc should be used with more cautious as it needs further investigation. ACE2 acts like carboxypeptidase and it could be inactivated through metalloprotease chelating. According to similarity between the action of ACE2 and carboxypeptidase, it is proposed that using chelating agent could control the COVID-19 host-cell-receptor (ACE2) and could have a step in controlling the disease.

\section{Conclusion}

COVID-19 is an enveloped virus with a positive single-stranded RNA. It constitutes outer wrapping or envelope that comes from the host cell rough endoplasmic reticulum (RER). COVID-19 genome has four structural proteins such as spike (S), envelope (E), nucleocapsid $(\mathrm{N})$, and membrane $(\mathrm{M})$ which the S protein is responsible to attach virus to host cell. The S protein consists RBD that binds virus to its receptor that is ACE2 on host cell. ACE2 has an extracellular domain 
that contains a single catalytic metallopeptidase unit that mostly consists metal ion on its active site which is mostly zinc. It has been suggested that zinc could enhance ACE2 activity and consequently increase the activity of COVID-19. This outcome needs further investigation while treating patients.

\section{References}

1. LiX, Geng M, Peng Y, Meng L, Lu S (2020) Molecular immune pathogenesis and diagnosis of COVID-19. J Pharm Anal 10(2): 102-108.

2. Lu R, Zhao X, Li J, Niu P, Yang B, et al. (2020) Genomic characterisation and epidemiology of 2019 novel coronavirus: implications for virus origins and receptor binding. The Lancet 395(10224): 565-574.

3. Fung TS, Liu DX (2014) Coronavirus infection, ER stress, apoptosis and innate immunity. Front Microbiol 5(296).

4. Tai W, He L, Zhang X, Pu J, Voronin D, et al. (2020) Characterization of the receptor-binding domain (RBD) of 2019 novel coronavirus: implication for development of RBD protein as a viral attachment inhibitor and vaccine. Cell Mol Immunol 17(6): 613-620.

5. Inoue T, Tsai B (2013) How viruses use the endoplasmic reticulum for entry, replication, and assembly. Cold Spring Harb Perspec Biol 5(1): a013250.

6. Almanza A, Carlesso A, Chintha C, Creedican S, Doultsinos D, et al.(2019) Endoplasmic reticulum stress signalling - from basic mechanisms to clinical applications. FEBS J 286(2): 241-278.

7. De Gascun CF, Carr MJ (2013) Human polyomavirus reactivation: disease pathogenesis and treatment approaches. Clin Dev Immunol 2013: 373579.

8. Darling NJ, Cook SJ (2014) The role of MAPK signalling pathways in the response to endoplasmic reticulum stress. Biochimica et Biophysica Acta (BBA)-Molecular Cell Res 1843(10): 2150-2163.

\section{ISSN: 2574-1241}

DOI: 10.26717/BJSTR.2020.30.005007

Sivakumar J T Gowder. Biomed J Sci \& Tech Res

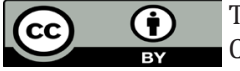

This work is licensed under Creative Commons Attribution 4.0 License

Submission Link: https://biomedres.us/submit-manuscript.php
9. Zheng M, Song L (2020) Novel antibody epitopes dominate the antigenicity of spike glycoprotein in SARS-CoV-2 compared to SARS-CoV. Cell Mol Immunol 17(5): 536-538.

10. Zhang H, Penninger JM, Li Y, Zhong N, Slutsky AS (2020) Angiotensinconverting enzyme 2 (ACE2) as a SARS-CoV-2 receptor: molecular mechanisms and potential therapeutic target. Intensive Care Med 46(4): 586-590.

11. Tikellis C, Thomas MC (2012) Angiotensin-converting enzyme 2 (ACE2) is a key modulator of the renin angiotensin system in health and disease. International Journal of Peptides 2012: 256294.

12. (2020) Database NCfBIP. Angiotensin II.

13. Kuster GM, Pfister O, Burkard T, Zhou Q, Twerenbold R, et al. (2020) SARS-CoV2: should inhibitors of the renin-angiotensin system be withdrawn in patients with COVID-19? Eur Heart J 41(19): 1801-1803.

14. Cerdà Costa N, Xavier Gomis Rüth F (2014) Architecture and function of metallopeptidase catalytic domains. Protein Science 23(2): 123-144.

15. Rawlings ND, Salvesan G (2013) Handbook of proteolytic enzymes 2013 : ISBN 012382219X, 9780123822192.

16. Guy JL, Lambert DW, Warner FJ, Hooper NM, Turner AJ (2005) Membrane-associated zinc peptidase families: comparing ACE and ACE2. Biochim Biophys Acta 1751(1): 2-8.

17. Chen Z, Huang S, Zhao M (2016) Molecularly imprinted polymers for biomimetic catalysts. Molecularly Imprinted Catalysts: Elsevier pp. 229239.

18. Hojyo S, Fukada T (2016) Roles of Zinc Signaling in the Immune System. J Immunol Res 2016: 6762343.

$\begin{array}{ll}\text { BIOMEDICAL } & \text { Assets of Publishing with us } \\ \text { RESEARCHES } & \text { - Global archiving of articles } \\ & \text { - Immediate, unrestricted online access } \\ & \text { - Rigorous Peer Review Process } \\ & \text { - Authors Retain Copyrights }\end{array}$

